\title{
Note on Language and Orthography
}

The Lese and the Efe consider themselves to speak mutually intelligible languages: talu Des-ba (the language of the Lese) and talu Efe-ba (the language of the Efe). Both of these are Sudanic languages. I shall refer to them simply as Lese and Efe. Most Lese and Efe men also speak some KiNgwana, a dialect of Swahili, and Lingala, two of the national languages of Zaire. A few Lese men know basic French. At the villages of Malembi, where this study was conducted, the younger Lese men are able to read and write KiNgwana and Lingala, but to my knowledge, few Lese women, and no Efe men or women, are able to read or write these languages. The Lese do not write their native language.

Except where noted, all transcriptions of narrative or terminology are reproduced here in the Lese language and translated into English. I have represented the sounds of Lese terms phonetically in romanized form but have dispensed with tonal markers and other diacritical notation. As no grammar of the Lese or Efe languages has been completed, I leave the difficult task of constructing a proper orthography and phonology to trained linguists.

I note some special pronunciations of Lese consonants: /b/ and /gb/ are implosive; $/ \mathrm{v} /$ is also implosive and lies midway between the English $/ \mathrm{b} /$ and $/ \mathrm{v} /$; finally, $/ \mathrm{l} /$ is a lateral flap similar to the sound of $a$ rolled $/ \mathrm{r} /$ or $/ \mathrm{d} \mathrm{l} /$.

Some Lese vowels are different from the vowels used in English or Swahili. However, all those vowels represented here are nearly identical in sound to the vowels $/ \mathrm{o} /, / \mathrm{i} /, / \mathrm{u} /, / \mathrm{a} /$, and $/ \mathrm{e} /$, as identified in the Interna- 
xviii

tional Phonetic Alphabet. Long vowels are indicated by a double vowel, for example, /aa/ or /ee/. When /i/ and /a/ appear as /ia/, the sound produced resembles the English semi-vowel /y/. The distinction between /o/ as in "lost" and "go" is not rendered.

All foreign words are italicized in the first use.

All individuals, clans, and phraties have been given pseudonymns. 